\title{
BEM-FEM coupling model for the dynamic analysis of piles and pile groups *
}

\author{
L.A. Padrón, J.J. Aznárez, O.Maeso \\ Instituto Universitario de Sistemas Inteligentes y Aplicaciones Numéricas en Ingeniería \\ (SIANI) Universidad de Las Palmas de Gran Canaria \\ Edificio Central del Parque Científico y Tecnológico \\ Campus Universitario de Tafira, 35017, Las Palmas de Gran Canaria, Spain \\ \{lpadron,jjaznarez,omaeso\}@siani.es
}

1 september 2006

\begin{abstract}
This paper shows a BEM-FEM coupling model for the time harmonic dynamic analysis of piles and pile groups embedded in an elastic half-space. Piles are modelled using Finite Elements (FEM) as a beam according to the Bernoulli hypothesis, while the soil is modelled using Boundary Elements (BEM) as a continuum, semi-infinite, isotropic, homogeneous or zoned homogeneous, linear, viscoelastic medium. It is assumed that the soil continuity is not altered by the presence of the piles, and the tractions at the pile-soil interface are considered as a load applied within the half-space. The formulation is exposed in detail. In order to validate the model, selected numerical results of time harmonic impedances of different pile groups configurations are evaluated and contrasted with other reference values taken from the literature.
\end{abstract}

\section{Introduction}

The frequency domain dynamic analysis of piles and pile groups embedded in a half-space has been treated numerically by several authors using different boundary integral formulations for the soil, and FEM (Finite Elements Method) for the piles, considered as monodimensional beam elements [1, $2,3,4,5,6,7,8,9]$. Also, the coupling of framed structures approached by FEM with threedimensional bodies represented by BEM (Boundary Elements Method) in time domain has been presented in $[10,11]$, where piles would be approximated using a special cylindrical boundary element.

Using BEM for both soil and piles, more versatile and rigorous numerical models have been developed: vibration isolation by a row of piles has been analyzed in $[12,13]$ and dynamic impedances of pile groups has been studied by two of the authors in $[14,15]$. High computational cost is a disadvantage of these models.

The present paper, in order to reduce the number of degrees of freedom in the problem, presents a different BEM-FEM model for the time harmonic dynamic analysis of piles and pile groups embedded in an elastic half-space, taking advantage of the particular characteristics of each one of the methods. This model is based on the idea of a previous static model developed in $[16,17,18]$

${ }^{*}$ This is the peer reviewed version of the following article: L.A. Padrón, J.J. Aznárez, O.Maeso, BEM-FEM coupling model for the dynamic analysis of piles and pile groups, Engineering Analysis with Boundary Elements 31 (2007) 473484, which has been published in final form at doi:10.1016/j.enganabound.2006.11.001. This work is released with a Creative Commons Attribution Non-Commercial No Derivatives License. 
where it is assumed that the continuity of the soil is not altered by the presence of the piles and where the tractions in the pile-soil interface are considered as a load applied within the half-space in the boundary integral representation of the soil.

Piles are modelled using FEM as beams according to the Bernoulli hypothesis, while soil is modelled using BEM as a continuum, semi-infinite, isotropic, homogeneous or zoned homogeneous, linear, viscoelastic medium. Welded boundary contact condition at the pile-soil interfaces are assumed. The formulation allows the analysis of problems including soil strata, rigid rocky beds and any topography for the soil surface. Furthermore, as the pile boundary does not need to be discretized, low computing times and memory requirements are needed. First, the formulation will be exposed in detail. After, in order to validate the model, numerical results for vertical, horizontal and rocking impedances are evaluated and compared to other reference values taken from the literature.

\section{Pile FE equations}

The behaviour of a pile submitted to dynamic loads can be described by the following differential equation

$$
\mathbf{M} \ddot{\mathbf{u}}(t)+\mathbf{C} \dot{\mathbf{u}}(t)+\mathbf{K} \mathbf{u}(t)=\mathbf{f}(t)
$$

where $\mathbf{M}, \mathbf{C}$ and $\mathbf{K}$ are the mass, damping and stiffness matrices of the pile, $\mathbf{u}(t)$ is the vector of nodal displacements and $\mathbf{f}(t)$ the vector of nodal forces over the pile.

It will be assumed now that the pile is subjected to a harmonically varying load. In this case, the vectors of nodal displacements and forces can be expressed as

$$
\mathbf{u}(t)=\mathbf{u}^{p} e^{\mathrm{i} \omega t} \quad ; \quad \mathbf{f}(t)=\mathbf{F} e^{\mathrm{i} \omega t}
$$

where $\mathbf{u}^{p}$ is the vector of nodal translations and rotations amplitudes, $\mathbf{F}$ is the vector of nodal forces amplitudes, $\omega$ the circular frequency of the excitation and $i=\sqrt{-1}$. Then, and considering a pile with zero internal damping, Eq.(1) becomes

$$
\left(\mathbf{K}-\omega^{2} \mathbf{M}\right) \mathbf{u}^{p}=\mathbf{F}
$$

Piles are modelled by FEM as vertical beams according to the Bernoulli hypothesis, and are discretized using a three-nodes element, shown in Fig. 1, that has been defined in order to be able to adjust the deformed shape accurately with a scarce number of elements. There are 13 degrees of freedom defined on it: two lateral displacements and a vertical displacement on each node, and two rotations $\theta$ on each one of the extreme nodes, one about $x_{1}$ axis and another one about $x_{2}$.

The lateral displacements $u_{1}$ and $u_{2}$ along the element are approximated by a set of fourth degree shape functions, while vertical displacements $u_{3}$ are approximated by one of second degree. Thus

$$
\begin{gathered}
u_{i}=\varphi_{1} u_{k_{i}}+\varphi_{2} \theta_{k_{i}}+\varphi_{3} u_{l_{i}}+\varphi_{4} u_{m_{i}}+\varphi_{5} \theta_{m_{i}} \quad ; \quad i=1,2 \\
u_{3}=\phi_{1} u_{k_{3}}+\phi_{2} u_{l_{3}}+\phi_{3} u_{m_{3}}
\end{gathered}
$$

where 

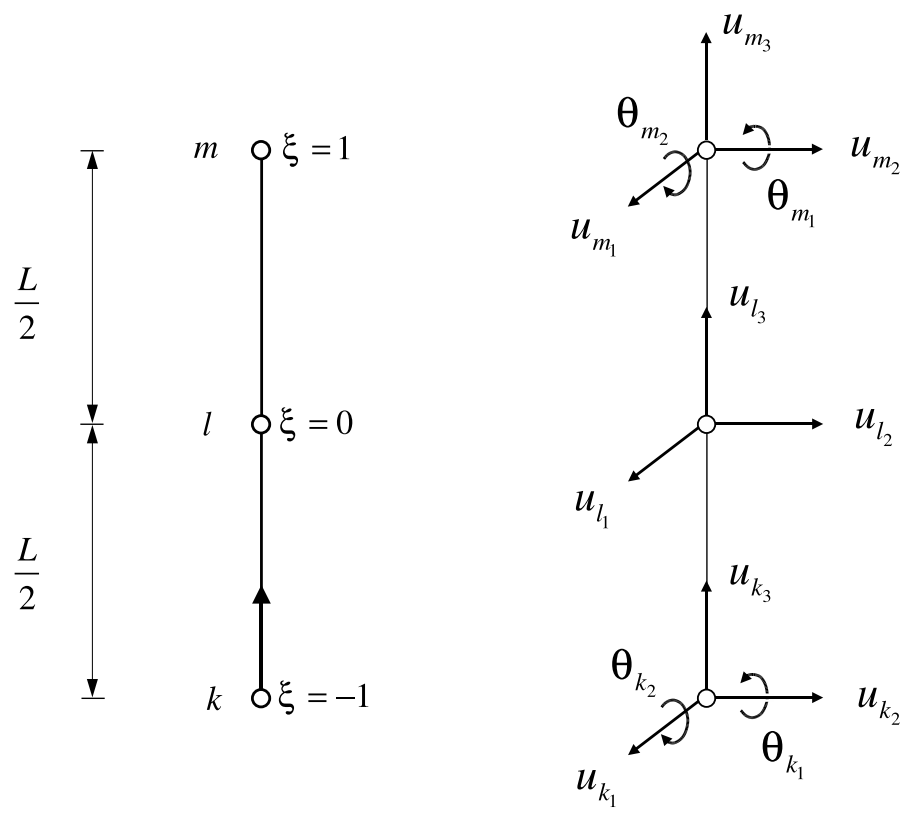

Figure 1: Finite element definition

$$
\begin{aligned}
\varphi_{1} & =\xi\left(-\frac{3}{4}+\xi+\frac{1}{4} \xi^{2}-\frac{1}{2} \xi^{3}\right) \\
\varphi_{2} & =\frac{1}{4} \xi\left(-1+\xi+\xi^{2}-\xi^{3}\right) \\
\varphi_{3} & =1-2 \xi^{2}+\xi^{4} \\
\varphi_{4} & =\xi\left(\frac{3}{4}+\xi-\frac{1}{4} \xi^{2}-\frac{1}{2} \xi^{3}\right) \\
\varphi_{5} & =\frac{1}{4} \xi\left(-1-\xi+\xi^{2}+\xi^{3}\right)
\end{aligned}
$$

and

$$
\begin{aligned}
\phi_{1} & =\frac{1}{2} \xi(\xi-1) \\
\phi_{2} & =1-\xi^{2} \\
\phi_{3} & =\frac{1}{2} \xi(\xi+1)
\end{aligned}
$$

being $\xi$ the elemental dimensionless coordinate varying from -1 to +1 .

Using the principle of virtual displacements and the shape functions defined above, the stiffness sub-matrix for the lateral behaviour of this element (denoted by super-index $l$ ) can be obtained as (see e.g. reference [19])

$$
k_{i j}^{l}=\int_{L} \varphi_{i}^{\prime \prime} E I \varphi_{j}^{\prime \prime} d x_{3} \quad ; \quad i, j=1, \ldots, 5
$$

and the one for the axial behaviour (denoted by super-index $a$ ) as

$$
k_{i j}^{a}=\int_{L} \phi_{i}^{\prime} E A \phi_{j}^{\prime} d x_{3} \quad ; \quad i, j=1,2,3
$$


where $E$ is the Young's Modulus for the pile, $A$ and $I$ are the area and the moment of inertia of the section of the pile and $L$ is the element length. Here, super-indexes ' denote derivative referring to $x_{3}$ coordinate.

Finally, the matrices obtained are

$$
\left(\begin{array}{c}
f_{k_{i}} \\
m_{k_{i}} \\
f_{l_{i}} \\
f_{m_{i}} \\
m_{m_{i}}
\end{array}\right)=\frac{E I}{5 L}\left[\begin{array}{ccccc}
\frac{316}{L^{2}} & \frac{94}{L} & \frac{-512}{L^{2}} & \frac{196}{L^{2}} & \frac{-34}{L} \\
\frac{94}{L} & 36 & \frac{-128}{L} & \frac{34}{L} & -6 \\
\frac{-512}{L^{2}} & \frac{-128}{L} & \frac{1024}{L^{2}} & \frac{-512}{L^{2}} & \frac{128}{L} \\
\frac{196}{L^{2}} & \frac{34}{L} & \frac{-512}{L^{2}} & \frac{316}{L^{2}} & \frac{-94}{L} \\
\frac{-34}{L} & -6 & \frac{128}{L} & \frac{-94}{L} & 36
\end{array}\right]\left(\begin{array}{c}
u_{k_{i}} \\
\theta_{k_{i}} \\
u_{l_{i}} \\
u_{m_{i}} \\
\theta_{m_{i}}
\end{array}\right) \quad ; \quad i=1,2
$$

and

$$
\left(\begin{array}{c}
f_{k_{3}} \\
f_{l_{3}} \\
f_{m_{3}}
\end{array}\right)=\frac{E A}{3 L}\left[\begin{array}{ccc}
7 & -8 & 1 \\
-8 & 16 & -8 \\
1 & -8 & 7
\end{array}\right]\left(\begin{array}{c}
u_{k_{3}} \\
u_{l_{3}} \\
u_{m_{3}}
\end{array}\right)
$$

Similarly, the mass influence coefficients for an element, that represents the inertia force opposing the acceleration experimented by a certain degree of freedom, can be evaluated by a similar procedure as

$$
m_{i j}^{l}=\int_{L} \varphi_{i} \bar{m} \varphi_{j} d x_{3} \quad ; \quad m_{i j}^{a}=\int_{L} \phi_{i} \bar{m} \phi_{j} d x_{3}
$$

Using the same functions that were used for calculating the stiffness matrix, the result obtained is the consistent-mass matrix. Thus, considering a beam with uniformly distributed mass $\bar{m}$, the matrices obtained for the lateral and axial behaviours are, respectively

$$
\mathbf{M}^{l}=L \bar{m}\left[\begin{array}{ccccc}
\frac{13}{63} & \frac{L}{63} & \frac{4}{63} & \frac{-23}{630} & \frac{L}{180} \\
\frac{L}{63} & \frac{L^{2}}{630} & \frac{2 L}{315} & \frac{-L}{180} & \frac{L^{2}}{1260} \\
\frac{4}{63} & \frac{2 L}{315} & \frac{128}{315} & \frac{4}{63} & \frac{-2 L}{315} \\
\frac{-23}{630} & \frac{-L}{180} & \frac{4}{63} & \frac{13}{63} & \frac{-L}{63} \\
\frac{L}{180} & \frac{L^{2}}{1260} & \frac{-2 L}{315} & \frac{-L}{63} & \frac{L^{2}}{630}
\end{array}\right] \quad ; \quad \mathbf{M}^{a}=\frac{L \bar{m}}{15}\left[\begin{array}{ccc}
2 & 1 & \frac{-1}{2} \\
1 & 8 & 1 \\
\frac{-1}{2} & 1 & 2
\end{array}\right]
$$

The considered forces acting over the pile are punctual forces and moments applied at the top of the pile and distributed forces arising from pile-soil interaction. An axial force at the tip of the pile has been taken into account also. So, the vector of nodal forces $\mathbf{F}$ can be decomposed as

$$
\mathbf{F}=\mathbf{F}^{e x t}+\mathbf{F}^{e q}=\mathbf{F}_{\text {top }}+\mathbf{F}_{p}+\mathbf{F}^{e q}
$$

where $\mathbf{F}^{e x t}$ include the forces at the top $\mathbf{F}_{\text {top }}$ and the axial force at the tip of the pile $\mathbf{F}_{p}$; and $\mathbf{F}^{e q}$ is the vector of the equivalent nodal forces from the pile-soil interaction, that can be calculated as

$$
\mathbf{F}^{e q}=\mathbf{Q} \cdot \mathbf{q}^{p}
$$

where $\mathbf{Q}$ is the matrix that transforms nodal force components to equivalent nodal forces.

The external forces defined over the generic element are schematized in Fig. 2. The tractions $\mathbf{q}^{p}$ along the pile-soil interface are approximated by the set of shape functions defined by Eq.(7) as 

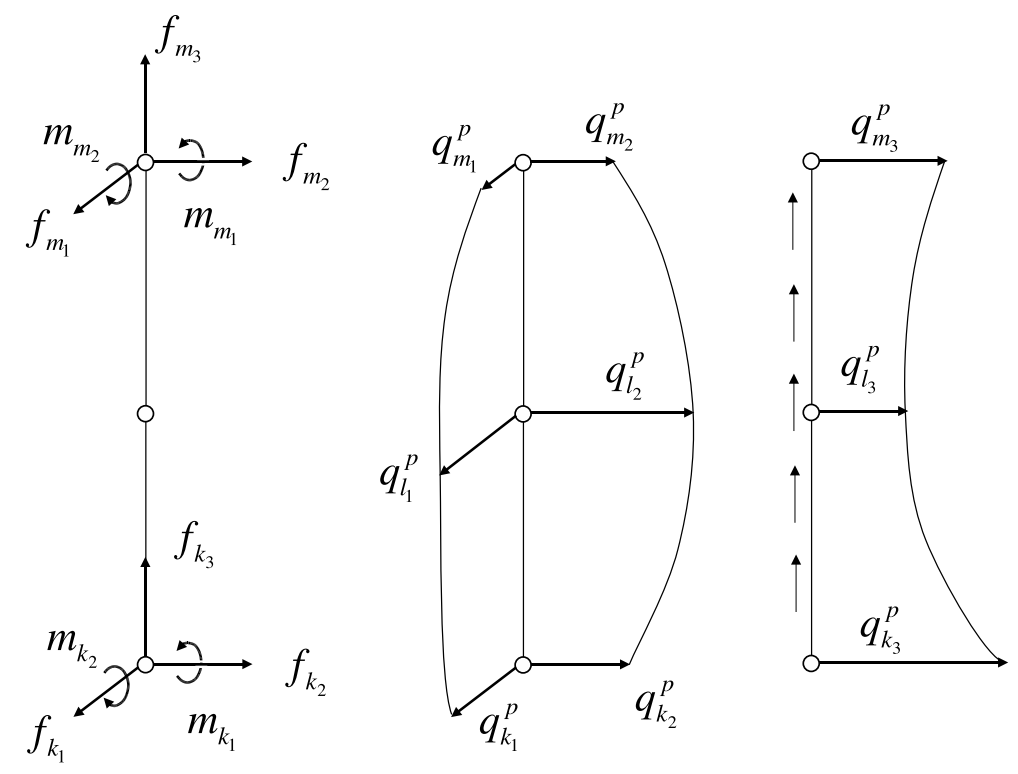

Figure 2: External punctual forces (left) and tractions along the pile-soil interface defined on the generic element

$$
q_{i}=\phi_{1} q_{k_{i}}+\phi_{2} q_{l_{i}}+\phi_{3} q_{m_{i}} \quad ; \quad i=1,2,3
$$

Again, using the principle of virtual displacements, the coefficients of matrix $\mathbf{Q}$ for lateral forces can be obtained as

$$
q_{i j}^{l}=\int_{L} \varphi_{i} \phi_{j} d x \quad ; \quad i=1, \ldots, 5 ; j=1,2,3
$$

and the ones for axial forces as

$$
q_{i j}^{a}=\int_{L} \phi_{i} \phi_{j} d x \quad ; \quad i, j=1,2,3
$$

This way, one can obtain the following matrices for lateral and axial equivalent nodal forces respectively

$$
\left(\begin{array}{c}
f_{k_{i}}^{e q} \\
m_{k_{i}}^{e q} \\
f_{l_{i}}^{e q} \\
f_{m_{i}}^{e q} \\
m_{m_{i}}^{e q}
\end{array}\right)=\left[\begin{array}{ccc}
\frac{23 L}{140} & \frac{11 L}{105} & \frac{-L}{28} \\
\frac{L^{2}}{84} & \frac{L^{2}}{105} & \frac{-L^{2}}{210} \\
\frac{4 L}{105} & \frac{16 L}{35} & \frac{4 L}{105} \\
\frac{-L}{28} & \frac{11 L}{105} & \frac{23 L}{140} \\
\frac{L^{2}}{210} & \frac{-L^{2}}{105} & \frac{-L^{2}}{84}
\end{array}\right]\left(\begin{array}{c}
q_{k_{i}}^{p} \\
q_{l_{i}}^{p} \\
q_{m_{i}}^{p}
\end{array}\right) \quad ; \quad i=1,2
$$

and

$$
\left(\begin{array}{c}
f_{k_{3}}^{e q} \\
f_{l_{3}}^{e q} \\
f_{m_{3}}^{e q}
\end{array}\right)=\frac{L}{30}\left[\begin{array}{ccc}
4 & 2 & -1 \\
2 & 16 & 2 \\
-1 & 2 & 4
\end{array}\right]\left(\begin{array}{c}
q_{k_{3}}^{p} \\
q_{l_{3}}^{p} \\
q_{m_{3}}^{p}
\end{array}\right)
$$


Once all elemental matrices have been obtained for the whole pile, one can write, for each one, the following expression

$$
\overline{\mathbf{K}} \mathbf{u}^{p}=\mathbf{F}^{e x t}+\mathbf{Q} \mathbf{q}^{p}
$$

where $\overline{\mathbf{K}}=\mathbf{K}-\omega^{2} \mathbf{M}$. As each pile will be discretized using as many elements as necessary to follow its deformed shape accurately, matrices $\overline{\mathbf{K}}$ and $\mathbf{Q}$ are global matrices, obtained as usual from the elemental ones.

\section{Soil BE equations}

The soil is modelled by BEM as a linear homogeneous isotropic viscoelastic unbounded region with complex valued shear modulus $\mu$ of the type $\mu=\operatorname{Re}[\mu](1+2 \mathrm{i} \xi)$, where $\xi$ is the damping coefficient. The boundary integral equation for a time-harmonic elastodynamic state defined in the domain $\Omega$ with boundary $\Gamma$ can be written in a condensed and general form as

$$
\mathbf{c}^{k} \mathbf{u}^{k}+\int_{\Gamma} \mathbf{p}^{*} \mathbf{u} d \Gamma=\int_{\Gamma} \mathbf{u}^{*} \mathbf{p} d \Gamma+\int_{\Omega} \mathbf{u}^{*} \mathbf{X} d \Omega
$$

where $\mathbf{c}^{k}$ is the local free term matrix at collocation point ' $k$ ', which is a diagonal matrix with a 1 on rows corresponding to internal points and a 0.5 on rows corresponding to boundary points where the boundary is smooth; $\mathbf{X}$ are the body forces in the domain $\Omega ; \mathbf{u}$ and $\mathbf{p}$ are the displacement and traction vectors; and $\mathbf{u}^{*}$ and $\mathbf{p}^{*}$ are the elastodynamic fundamental solution tensors on the boundary $\Gamma$ due to a time-harmonic concentrated load at point ' $k$ ':

$$
\mathbf{u}=\left\{\begin{array}{l}
u_{1} \\
u_{2} \\
u_{3}
\end{array}\right\} ; \quad \mathbf{p}=\left\{\begin{array}{c}
t_{1} \\
t_{2} \\
t_{3}
\end{array}\right\} ; \quad \mathbf{u}^{*}=\left[\begin{array}{ccc}
u_{11}^{*} & u_{12}^{*} & u_{13}^{*} \\
u_{21}^{*} & u_{22}^{*} & u_{23}^{*} \\
u_{31}^{*} & u_{32}^{*} & u_{33}^{*}
\end{array}\right] ; \quad \mathbf{p}^{*}=\left[\begin{array}{ccc}
t_{11}^{*} & t_{12}^{*} & t_{13}^{*} \\
t_{21}^{*} & t_{22}^{*} & t_{23}^{*} \\
t_{31}^{*} & t_{32}^{*} & t_{33}^{*}
\end{array}\right]
$$

The fundamental solution used in this work corresponds to the complete space because there is no closed form expression for the half-space fundamental solution, which depends on boundless integrals and would require approximate procedures for its evaluation. Therefore, the soil free surface should be discretized. In practice, however, only a small region around the foundation has to be included in the model to reach accurate results.

Generally, body forces $\mathbf{X}$ are considered to be zero in most of elastodynamic problems. Nevertheless, in this work, pile-soil interaction is replaced by internal punctual forces at the piles tip and load-lines placed along the piles axis, while it is assumed that the soil continuity is not altered by the presence of the piles, which is the main hypothesis that supports this model. The load-lines $\mathbf{q}^{s_{j}}$ within the soil and its relationship with tractions $\mathbf{q}^{p}$ are represented in Fig. 3 together with the internal punctual forces $F_{p_{j}}$ at the tip of the piles.

Then, Eq.(22) can be written as

$$
\mathbf{c}^{k} \mathbf{u}^{k}+\int_{\Gamma} \mathbf{p}^{*} \mathbf{u} d \Gamma=\int_{\Gamma} \mathbf{u}^{*} \mathbf{p} d \Gamma+\sum_{j=1}^{n_{p}}\left[\int_{\Gamma_{p_{j}}} \mathbf{u}^{*} \mathbf{q}^{s_{j}} d \Gamma_{p_{j}}-\Upsilon_{k}^{j} F_{p_{j}}\right]
$$

where $\Gamma_{p_{j}}$ is the pile-soil interface of pile $j, n_{p}$ is the total number of piles and $\Upsilon_{k}^{j}$ is a 3 components vector that represents the contribution of the axial force $F_{p_{j}}$ at the tip of the $j^{\text {th }}$ pile, when the concentrated load is applied on point ' $k$ '.

The boundary surface $\Gamma$ is discretized into quadratic elements of triangular and quadrilateral shapes with six and nine nodes, respectively (see reference [20]). Once the boundary has been 


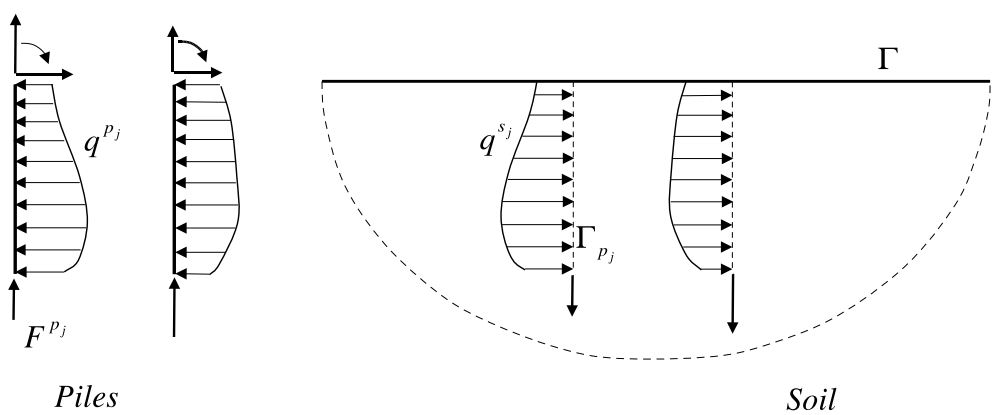

Figure 3: Load-lines representation.

discretized and Eq. (24) has been written for all nodes in $\Gamma$, the equation can be expressed in matrix form as

$$
\mathbf{H}^{s s} \mathbf{u}^{s}=\mathbf{G}^{s s} \mathbf{p}+\sum_{j=1}^{n_{p}} \mathbf{G}^{s p_{j}} \mathbf{q}^{s_{j}}-\sum_{j=1}^{n_{p}} \Upsilon^{s j} F_{p_{j}}
$$

where $\mathbf{u}^{s}$ is the vector of nodal displacements on the surface, $\mathbf{H}^{s s}$ and $\mathbf{G}^{s s}$ are matrices obtained by integration over $\Gamma$ of the 3-D elastodynamic fundamental solution times the shape functions of the boundary elements, and $\mathbf{G}^{s p_{j}}$ is the matrix obtained by integration over $\Gamma_{p_{j}}$ of the 3-D elastodynamic fundamental solution times the interpolation functions defined in (7), when the unit load is applied over $\Gamma$. Assuming free traction surface $(\mathbf{p}=0)$, Eq. (25) becomes

$$
\mathbf{H}^{s s} \mathbf{u}^{s}-\sum_{j=1}^{n_{p}} \mathbf{G}^{s p_{j}} \mathbf{q}^{s_{j}}+\sum_{j=1}^{n_{p}} \Upsilon^{s j} F_{p_{j}}=0
$$

Furthermore, Eq. (24) will be also applied on the piles internal nodes, so, for a certain pile $i$, one can write

$$
\mathbf{u}_{k}^{p_{i}}+\mathbf{H}^{p_{i} s} \mathbf{u}^{s}-\sum_{j=1}^{n_{p}} \mathbf{G}^{p_{i} p_{j}} \mathbf{q}^{s_{j}}+\sum_{j=1}^{n_{p}} \Upsilon^{p_{i} j} F_{p_{j}}=0
$$

where $\mathbf{u}_{k}^{p_{i}}$ is the vector of nodal displacements at the node $k$ of the pile $i$ where the unit load is applied, $\mathbf{H}^{p_{i} s}$ is the matrix obtained by integration over $\Gamma$ of the 3-D elastodynamic fundamental solution times the shape functions of the boundary elements, and $\mathbf{G}^{p_{i} p_{j}}$ is the matrix obtained by integration over $\Gamma_{p_{j}}$ of the 3-D elastodynamic fundamental solution times the interpolation functions defined in (7), when the unit load is applied over a pile $i$.

Besides, as an axial force at the pile tip is considered, an extra equation needs to be written. To do so, the unit load must be applied in the $x_{3}$ direction at any no-nodal point. The point with elemental dimensionless coordinate $\xi=-0.5$ in the bottom element of the pile has been chosen because of its nearness to the tip. This way, the extra equation is

$$
\frac{1}{8}\left(3 u_{3}^{b_{k}}+6 u_{3}^{b_{l}}-u_{3}^{b_{m}}\right)+\int_{\Gamma} \hat{\mathbf{p}}^{*} \mathbf{u} d \Gamma_{s}=\sum_{j=1}^{n_{p}}\left[\int_{\Gamma_{p_{j}}} \hat{\mathbf{u}}^{*} \mathbf{q}^{s_{j}} d \Gamma_{p_{j}}-\left(\Upsilon_{b}^{j}\right)_{3} F_{p_{j}}\right]
$$

where $u_{3}^{b_{k}}, u_{3}^{b_{l}}$ and $u_{3}^{b_{m}}$ are the vertical displacements of nodes $k, l$ and $m$ of the bottom element, $\hat{\mathbf{p}}^{*}=\left\{p_{31}^{*}, p_{32}^{*}, p_{33}^{*}\right\}$ and $\hat{\mathbf{u}}^{*}=\left\{u_{31}^{*}, u_{32}^{*}, u_{33}^{*}\right\}$. In a matrix form, Eq. (28) can be written as 


$$
\mathbf{D} \mathbf{u}_{b}^{p_{i}}+\mathbf{H}_{e}^{p_{i} s} \mathbf{u}^{s}-\sum_{j=1}^{n_{p}} \mathbf{G}_{e}^{p_{i} p_{j}} \mathbf{q}^{s_{j}}+\sum_{j=1}^{n_{p}} \Upsilon_{b_{3}}^{p_{i j}} F_{p_{j}}=0
$$

where $\mathbf{u}_{b}^{p_{i}}$ is the vector of nodal displacements at the bottom element nodes of the pile $i$ where the unit load is applied, $\mathbf{H}_{e}^{p_{i} s}$ is a vector obtained by integration over $\Gamma$ of the 3 -D elastodynamic fundamental solution times the shape functions of the boundary elements, and $\mathbf{G}_{e}^{p_{i} p_{j}}$ is a vector obtained by integration over $\Gamma_{p_{j}}$ of the 3-D elastodynamic fundamental solution times the interpolation functions defined in (7), when the unit load is applied on the extra point of the pile $i$. $\mathbf{D}$ is the vector $1 / 8\{0,0,3,0,0,6,0,0,-1\}$.

The integrals of $\mathbf{u}^{*} \mathbf{q}^{s_{j}}$ in (24) and $\hat{\mathbf{u}}^{*} \mathbf{q}^{s_{j}}$ in (28) over $\Gamma_{p_{j}}$ are calculated as a monodimensional integral extended to a load-line, defined by the pile axis, when the collocation point is outside pile $j$. However, this integrals have a singularity at the collocation point when applied on the integrated pile. In this case, in order to avoid this singularity, the integrals are evaluated over a cylinder which radius $R_{p}$ is $\sqrt{\pi / A}$. This way, let us consider the pile-soil interface $\Gamma_{p}$ (whatever the kind of section) as a cylinder of radius $R_{p}$ where tractions $\sigma_{p s}$ are applied. The last term of Eq. (22) includes integrals of the type

$$
\int_{\Gamma_{p}} \sigma_{p s} \mathbf{u}^{*} d \Gamma_{p}=\int_{\Gamma_{p}} \frac{\mathbf{q}^{s}}{2 \pi R_{p}} \mathbf{u}^{*} d \Gamma_{p}=\frac{1}{2 \pi R_{p}} \sum_{e=1}^{N_{e}} \sum_{i=k, l, m} \mathbf{q}_{i}^{s} \int_{\Gamma_{p_{e}}} \phi_{i} \mathbf{u}^{*} d \Gamma_{p}
$$

where $N_{e}$ is the number of elements in which the load-line has been discretized and Eq. (16) has been used to express $\mathbf{q}^{s}$ along each element. The elastodynamic fundamental solution, that gives the displacement in a $k$ direction when the load is applied in the $l$ direction, can be written as

$$
\begin{gathered}
u_{l k}^{*}=\frac{1}{4 \pi \mu}\left[\psi \delta_{l k}-\chi r_{, k} r_{, l}\right] \\
\psi=-\left(\frac{c_{2}}{c_{1}}\right)^{2}\left(\frac{1}{z_{1}^{2} r^{2}}-\frac{1}{z_{1} r}\right) \frac{e^{z_{1} r}}{r}+\left(\frac{1}{z_{2}^{2} r^{2}}-\frac{1}{z_{2} r}+1\right) \frac{e^{z_{2} r}}{r} \\
\chi=-\left(\frac{c_{2}}{c_{1}}\right)^{2}\left(\frac{3}{z_{1}^{2} r^{2}}-\frac{3}{z_{1} r}+1\right) \frac{e^{z_{1} r}}{r}+\left(\frac{3}{z_{2}^{2} r^{2}}-\frac{3}{z_{2} r}+1\right) \frac{e^{z_{2} r}}{r}
\end{gathered}
$$

where $\mu$ is the shear modulus, $\delta_{l k}$ is the Kronecker delta function, $c_{1}$ and $c_{2}$ are the wave velocities for $\mathrm{P}$ and $\mathrm{S}$ waves and $z_{j}=-\mathrm{i} k_{j}$, being $k_{1}$ and $k_{2}$ the wave numbers for $\mathrm{P}$ and $\mathrm{S}$ waves, respectively.

Then, the integrals in the last term of Eq. (30) can be solved, in cylindrical coordinates (see Fig. 4), as

$$
\begin{array}{r}
\int_{\Gamma_{p_{e}}} \phi_{i} \mathbf{u}^{*} d \Gamma_{p}=\int_{x_{3}^{r}} \int_{\theta} \frac{1}{4 \pi \mu}\left[\psi \delta_{l k}-\chi r_{, k} r_{, l}\right] \phi_{i} R_{p} d \theta d x_{3}= \\
\frac{R_{p}}{4 \pi \mu} \int_{x_{3}^{r}} \phi_{i}\left[2 \pi \psi \delta_{l k}-\frac{\pi}{r^{2}} \chi \mathbf{R}_{l k}\right] d x_{3}
\end{array}
$$

where $x_{3}^{r}=x_{3}-x_{3}^{k}$ and

$$
\mathbf{R}_{l k}=\left(\begin{array}{ccc}
R_{p}^{2} & 0 & 0 \\
0 & R_{p}^{2} & 0 \\
0 & 0 & 2\left(x_{3}^{r}\right)^{2}
\end{array}\right)
$$




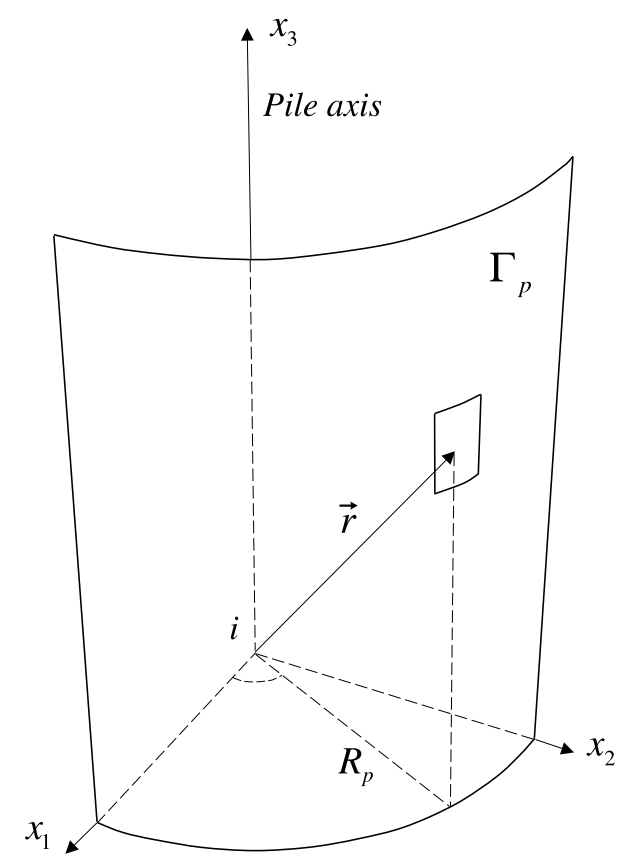

Figure 4: Integration over pile-soil interface when the collocation point belongs to the pile

Now, the integral of Eq. (30) can be written as

$$
\int_{\Gamma_{p}} \sigma_{p s} \mathbf{u}^{*} d \Gamma_{p}=\sum_{e=1}^{N_{e}} \frac{R_{p} L_{e}}{8 \mu} \sum_{i=k, l, m} \mathbf{q}_{j}^{s} \int_{-1}^{1} \phi_{i}\left[2 \psi \delta_{l k}-\frac{\chi}{r^{2}} \mathbf{R}_{l k}\right] d \xi
$$

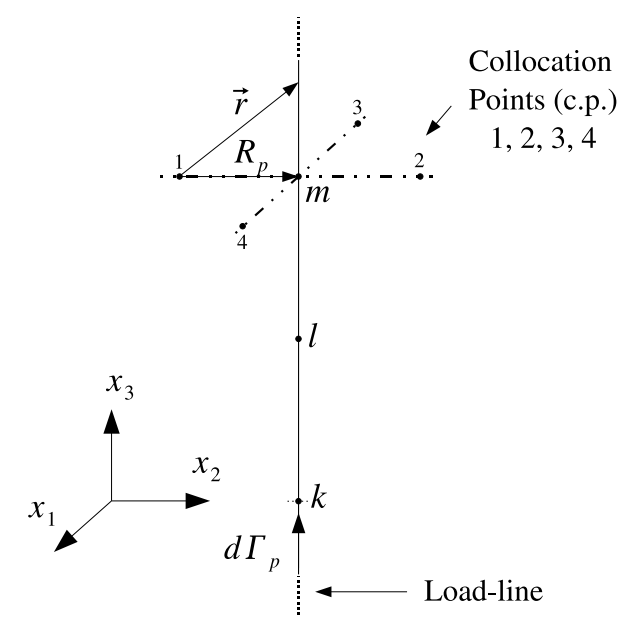

Figure 5: No-nodal collocation strategy

Nevertheless, to compute integrals over $\Gamma_{p_{j}}$ from the same pile, a no nodal collocation strategy could also be carried out. This will lead to a procedure that allows the reinterpretation of the previous equation. This way, in order to avoid breaking the problem symmetries, at least four collocation points, symmetrically placed around the pile, should be chosen (see Fig. 5). A single equation can be obtained by adding these four equations divided by four, so that the arising coefficients are of 
the same order. In this case, integrating over the load-line, the last term of Eq. (22) becomes

$$
\begin{aligned}
& \int_{\Gamma_{p}} \mathbf{q}^{s} \mathbf{u}^{*} d \Gamma_{p}=\frac{1}{4} \sum_{\substack{n=1 \\
c . p .}}^{4} \sum_{e=1}^{N_{e}} \sum_{i=k, l, m} \frac{\mathbf{q}_{i}^{s}}{4 \pi \mu} \int_{x_{3}^{r}} \phi_{i}\left[\psi \delta_{l k}-\chi_{, k} r_{, l}\right] d x_{3}^{r}= \\
& =\frac{1}{4} \sum_{\substack{n=1 \\
c . p .}}^{4} \sum_{e=1}^{N_{e}} \sum_{i=k, l, m} \frac{\mathbf{q}_{i}^{s}}{4 \pi \mu} \int_{x_{3}^{r}} \phi_{i}\left[\psi \delta_{l k}-\frac{\chi}{r^{2}} \mathbf{R}_{l k}^{n}\right] d x_{3}^{r}
\end{aligned}
$$

where

$$
\underset{n=1,2}{\mathbf{R}_{l k}^{n}}=\left(\begin{array}{ccc}
0 & 0 & 0 \\
0 & R_{p}^{2} & (-1)^{n} R_{p} x_{3}^{r} \\
0 & (-1)^{n} R_{p} x_{3}^{r} & \left(x_{3}^{r}\right)^{2}
\end{array}\right) \quad \underset{n=3,4}{\mathbf{R}_{l k}^{n}}=\left(\begin{array}{ccc}
R_{p}^{2} & 0 & (-1)^{n} R_{p} x_{3}^{r} \\
0 & 0 & 0 \\
(-1)^{n} R_{p} x_{3}^{r} & 0 & \left(x_{3}^{r}\right)^{2}
\end{array}\right)
$$

It can be seen that this procedure leads to an equation in each direction completely equivalent to Eq. (34), i.e., both methods are analogous.

On the other hand, when the collocation point ' $k$ ' is placed outside the pile $j$, one can write

$$
\Upsilon_{k}^{j}=\left\{\mathbf{u}_{13}^{*} \mathbf{u}_{23}^{*} \mathbf{u}_{33}^{*}\right\}_{k}
$$

However, when the collocation point is placed at the bottom node of pile $j, \Upsilon_{k}^{j} F_{p_{j}}$ becomes singular. In this case, to avoid this singularity, the axial force at the tip can be considered as a constant pressure $\sigma_{b}$ over a circular surface with radius $R_{p}=\sqrt{\pi / A}$. In this work, this strategy has been used whenever the collocation point is placed on the axis of pile $j$. According to this, one can write

$$
\Upsilon_{k}^{j} F_{p_{j}}=\int_{\Gamma_{b}} \mathbf{u}_{i 3}^{*} \sigma_{b} d \Gamma_{b}
$$

where $\Gamma_{b}$ is the tip surface and $\sigma_{b}=F_{p} / A$.

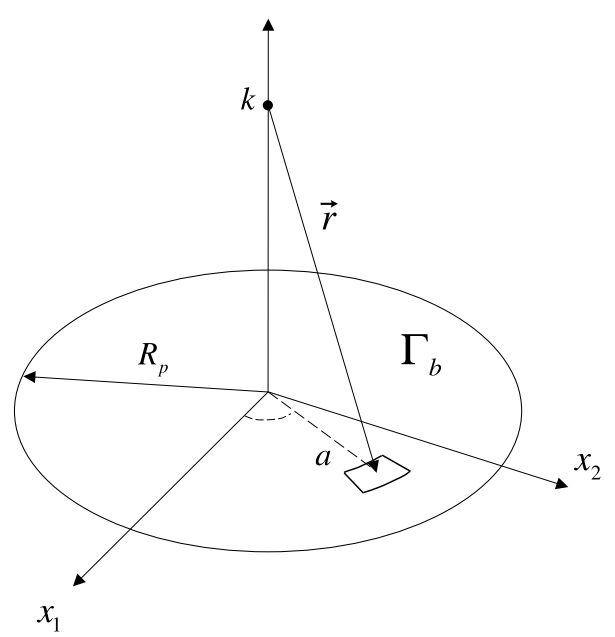

Figure 6: Integration over pile tip surface

Then, in cylindrical coordinates (see Fig. 6), Eq. (37) becomes 


$$
\Upsilon_{k}^{j}=\frac{1}{4 \pi \mu A} \int_{0}^{2 \pi} \int_{0}^{R_{p}}\left[\psi \delta_{i 3}-\chi_{, i} r_{, 3}\right] a d a d \theta=\frac{1}{2 \mu A} \int_{0}^{R_{p}}\left[\psi \delta_{i 3}-\left(x_{3}^{r}\right)^{2} \frac{\chi}{r^{2}}\right] a d a
$$

where the integral is regular and can be solved numerically.

Finally, all the integrals over $\Gamma$ are also solved numerically. Integrals of $O\left(\frac{1}{r}\right)$ are solved according to Li et al. [21]. Strongly-singular integrals are solved by identifying the $O\left(\frac{1}{r^{2}}\right)$ terms and splitting them up into a regular surface integral and a linear integral over the element perimeter. Then, singularities get cancelled with the contribution of adjacent elements. (see reference [22])

\section{BEM-FEM coupling equation}

Now, a global system of equations must be built using the expressions defined above. The links between piles and soil that will allow to do the coupling are the tractions $\mathbf{q}^{s}=-\mathbf{q}^{p}$ along the pile-soil interface and the displacements $\mathbf{u}^{p}$ along the pile.

As it is assumed that the soil continuity is not altered by the presence of the pile, the value of distributed mass assigned to the pile should be modified as $\bar{m}=A\left(\rho_{p}-\rho_{s}\right)$ so as not to overestimate the total mass introduced in the model, being $\rho_{p}$ and $\rho_{s}$ the pile and soil densities. A similar consideration was assumed in [1] and [2].

Eq. (21) becomes

$$
\overline{\mathbf{K}} \mathbf{u}^{p}-\mathbf{F}_{p}+\mathbf{Q} \mathbf{q}^{s}=\mathbf{F}_{t o p}
$$

Using equilibrium and compatibility conditions along the interface, and assuming the tractions $\mathbf{q}^{s}$ as positive, Eqs. (26), (27), (29) and (39) can be rearranged as

$$
\mathbf{A x}=\mathbf{B}
$$

where $\mathbf{B}$ is the right-hand vector when all external conditions have been applied,

$$
\begin{aligned}
& \mathbf{A}=
\end{aligned}
$$

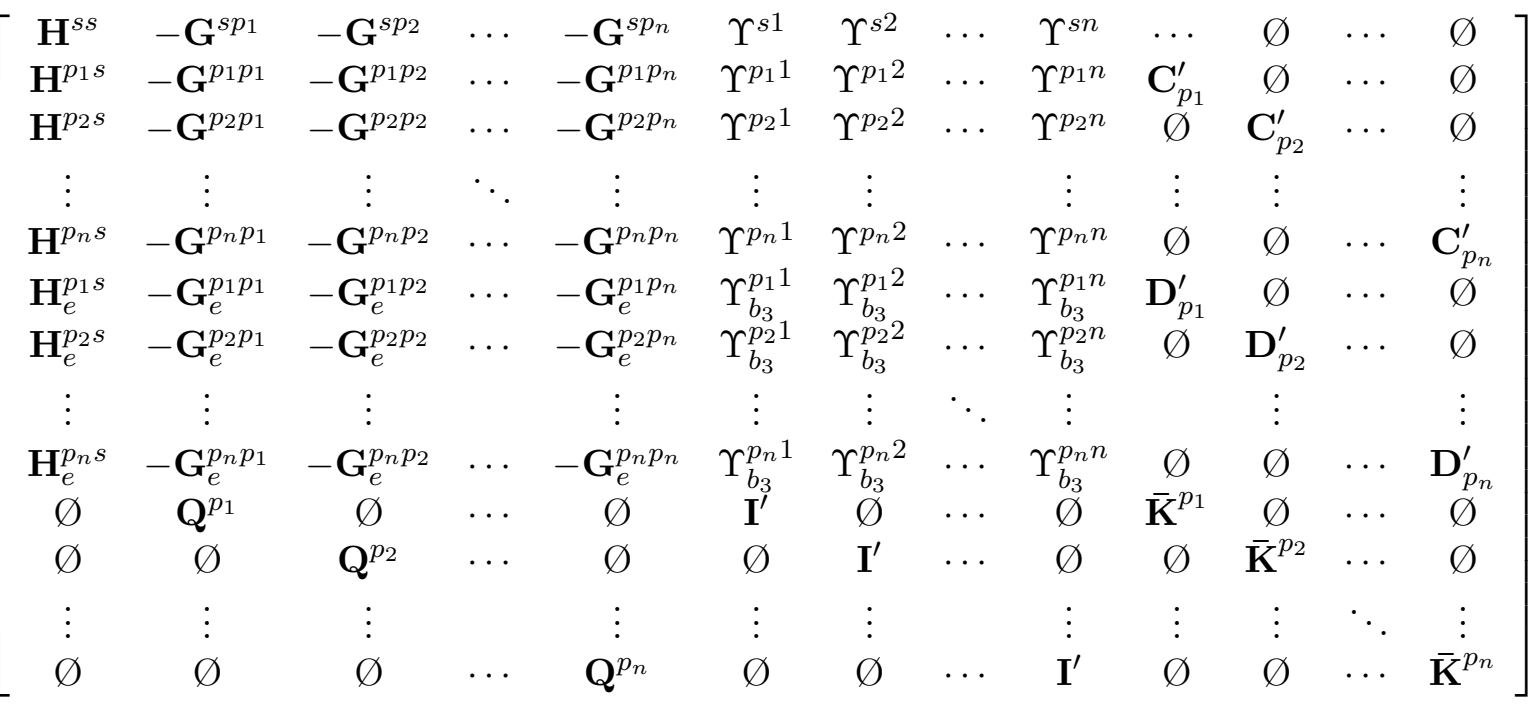

and

$$
\mathbf{x}=\left\{\mathbf{u}^{s}, \mathbf{q}^{s_{1}}, \mathbf{q}^{s_{2}}, \ldots, \mathbf{q}^{s_{n}}, F_{p_{1}}, F_{p_{2}}, \ldots, F_{p_{n}}, \mathbf{u}^{p_{1}}, \mathbf{u}^{p_{2}}, \ldots, \mathbf{u}^{p_{n}}\right\}^{T}
$$




\section{$5 \quad$ Validation results}

In order to validate the BEM-FEM coupling model, several results of impedances of pile groups have been computed and compared to other reference values taken from the literature.

The dynamic stiffness matrix $K_{i j}$ of a pile relates the vector of forces (and moments) applied at the pile top to the resulting vector of displacements (and rotations) at the same point. For a group of piles, it is assumed that the pile heads are constrained by a rigid pile-cap, and the foundation stiffness is the addition of the contributions of each pile. Fig. 7 illustrates the approached problem for a usual configuration, where $L$ and $d$ are used to denote the length and diameter of the piles, and $s$ refers to the distance between adjacent piles.
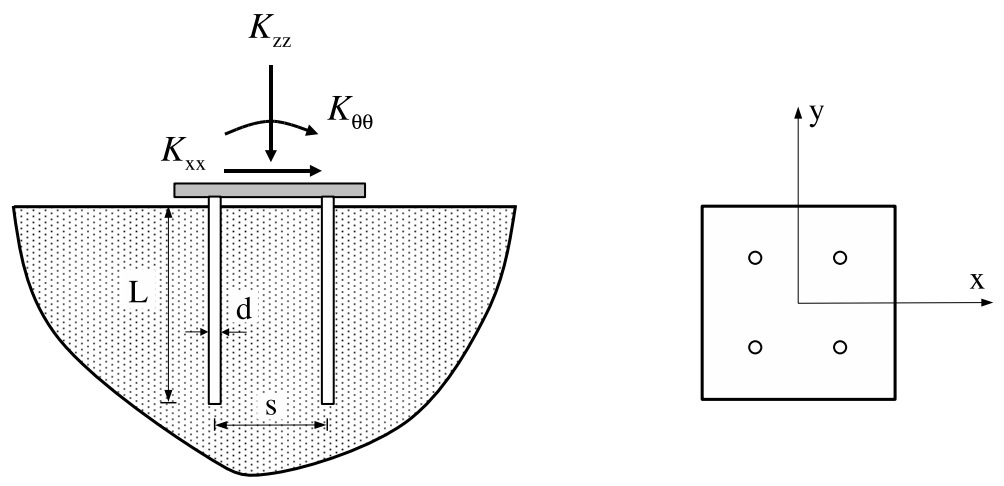

Figure 7: $2 \times 2$ pile group embedded in a half-space. Problem geometry definition.

The dynamic stiffness terms for a time harmonic excitation are functions of frequency $\omega$ and they are usually written as

$$
K_{i j}=k_{i j}+\mathrm{i} a_{o} c_{i j}
$$

where $k_{i j}$ and $c_{i j}$ are the frequency dependent dynamic stiffness and damping coefficients, respectively, $a_{o}$ is the dimensionless frequency

$$
a_{o}=\frac{\omega d}{c_{s}}
$$

and $c_{s}$ is the soil shear-wave velocity.

Once the problem has been solved for a unitary displacement applied at the cap, and the vectors $\mathbf{u}^{s}$ and $\mathbf{q}^{s}$ have been computed, the dynamic stiffness at a certain frequency can be easily obtained by applying Eq. (21) at the top element of each pile.

Fig. 8 shows a sketch of the discretizations used to obtain the stiffness of different pile groups embedded in a viscoelastic half-space, where boundary elements for the soil and mono-dimensional finite elements for the piles were used. As the developed software incorporates symmetry properties, only a quarter of the total geometry of the problem has to be discretized. Rectangular quadratic nine-nodes elements were used for the free surface. The length of free surface needed is, approximately, three times the pile length, and the element size is chosen in such a way that its main dimension is always shorter than the half of the wave length. On the other hand, the three-nodes elements defined above were used on the pile. Three equal elements for vertical problems and six elements, of which three concentrated on the top, for horizontal and rocking problems were used. It should be noted that the model allows to change the number and position of piles without modifying the free surface discretization. 


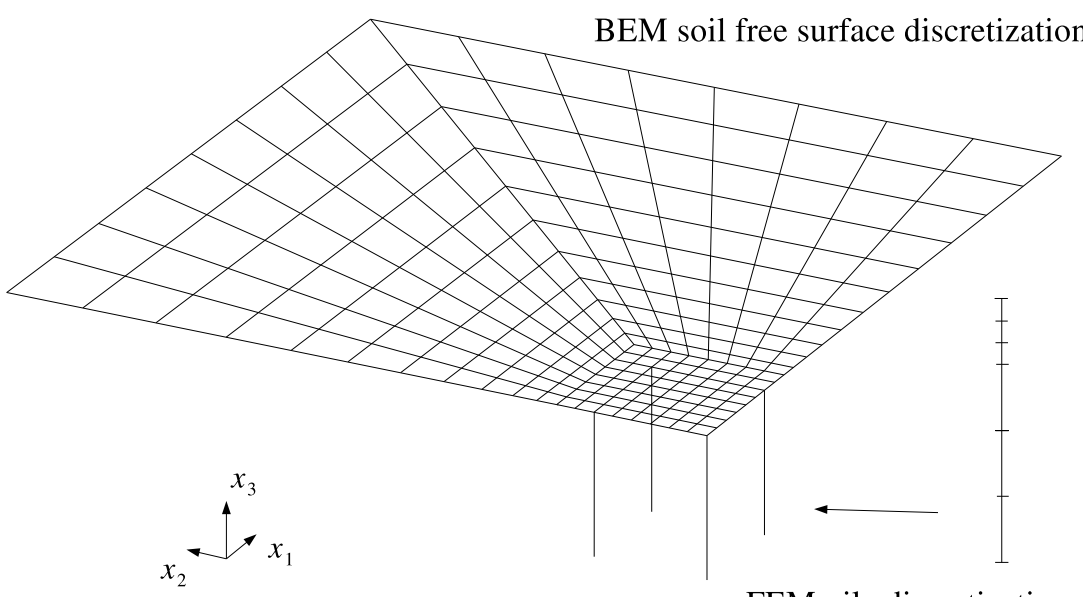

FEM pile discretization

Figure 8: 3x3 Pile group BEM-FEM discretization for horizontal problems

Three sets of results are presented in this section. In the first block, several results are compared with those of Kaynia [1], obtained from an analysis of single piles and pile groups considering piles as linear elastic prismatic members and soil as semi-infinite viscoelastic media by constructing the requisite Green's function using a discrete layer matrix approach. The following properties are taken from Kaynia [1]: piles (in the sequel denoted by sub-index p) are assumed to be elastic beams and surrounding soil a uniform viscoelastic media with internal damping coefficient $\beta=0.05$; ratio between densities $\rho_{s} / \rho_{p}=0.7$; and Poisson ratios $\nu_{s}=0.4$ (for the soil) and $\nu_{p}=0.25$ (for the pile, but not taken into account in the proposed technique). Two different soils with ratios between the Young modulus $E_{p} / E_{s}=10^{3}$ (soft soil) and $E_{p} / E_{s}=10^{2}$ (stiff soil) have been considered.

Lateral, vertical and rocking impedances (stiffness and damping coefficients) of $3 \times 3$ pile groups embedded in a homogeneous isotropic viscoelastic half-space, obtained by proposed technique (noted by BEM-FEM in the Figs.), are shown from Figs. 9 to 11, all for $s / d=2,5,10$, and soft soil. Lateral, vertical and rocking impedances of $2 \times 2,3 \times 3$ and $4 \times 4$ pile groups embedded in stiff soils are shown from Figs. 12 to 14 . The piles aspect ratio is $L / d=15$. The vertical and horizontal impedance functions have been normalized with respect to the respective single pile static stiffness $\left(k^{s}\right)$ times the number $(N)$ of piles in the group. The rocking impedances have been normalized with respect to the sum of the products of the respective single pile static stiffness $\left(k_{z z}^{s}\right)$ times the square of the distance to the rotation axis $\left(x_{i}\right)$. All results are plotted versus the dimensionless frequency parameter defined by Eq. (44).

In the second set, several results for more slender piles $(L / d=20)$ are compared with those of Miura et al. [8], where a 3-D formulation based on Green's functions of cylindrical loads in layered semi-infinite media is employed. The properties used are: soil internal damping coefficient $\beta=0.05$; ratio between the material modulae $E_{p} / E_{s}=10^{2}$; ratio between densities $\rho_{s} / \rho_{p}=0.7$; and Poisson's ratios $\nu_{s}=0.4$ (for the soil) and $\nu_{p}=0.25$. The piles ratio between pile separation and diameter is $s / d=5$.

Lateral and vertical stiffness and damping coefficients of $2 \times 2$ and $4 \times 4$ pile groups embedded in a homogeneous isotropic viscoelastic half-space are shown in Figs. 15 and 16.

Miura et al. also analyze the load distribution among the pile caps in $4 \times 4$ pile groups. In Fig. 17, the distribution of shear and axial forces due to horizontal and vertical excitation respectively are shown and compared with those given in [8].

All results from Kaynia and Miura et al. have been taken directly from [1] and [8] and may contain small errors. It can be seen that the computed values are in very good agreement with 

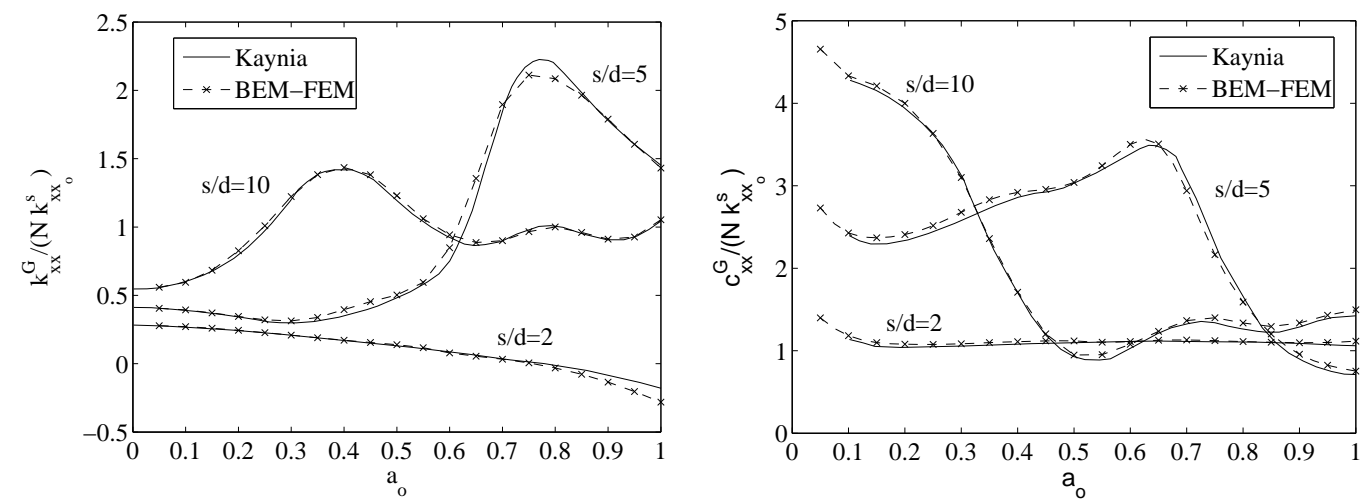

Figure 9: Horizontal impedances of 3x3 pile groups in soft soil. Comparison with Kaynia's solution.
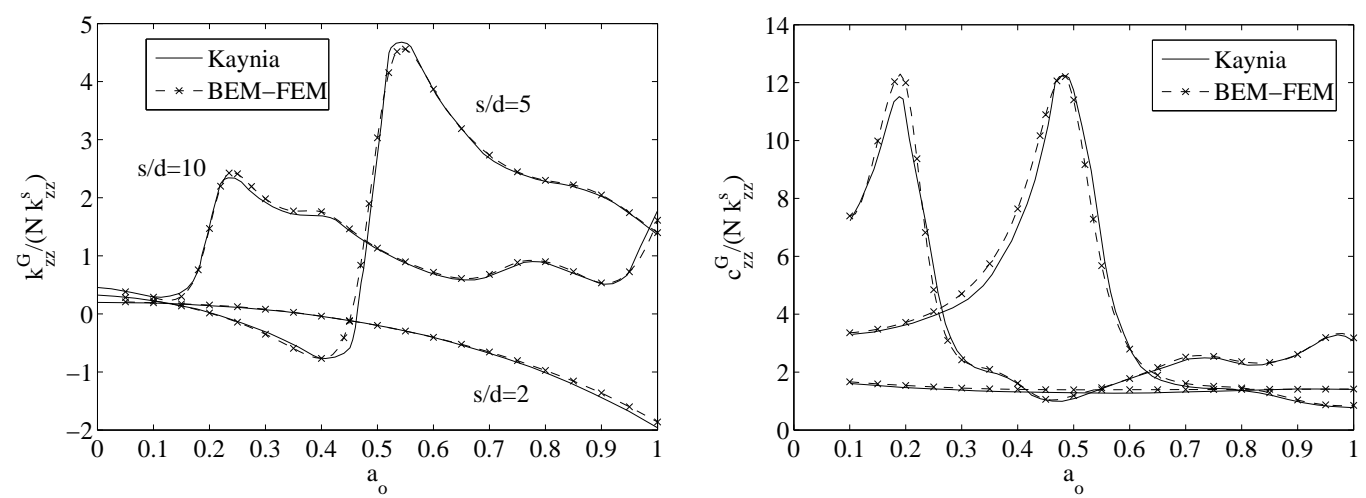

Figure 10: Vertical impedances of 3x3 pile groups in soft soil. Comparison with Kaynia's solution.
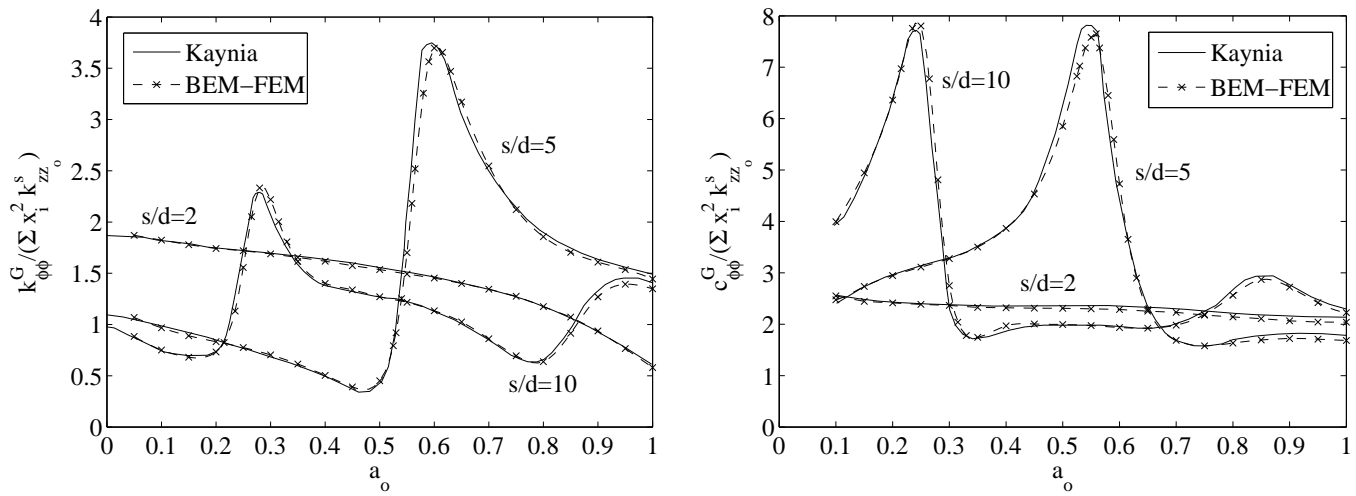

Figure 11: Rocking impedances of 3x3 pile groups in soft soil. Comparison with Kaynia's solution.

those presented in the respective cites. It should be noted that the results computed using the BEM-FEM model proposed here are also very close to others (not shown in this paper for the sake of brevity) obtained using a more rigorous 3D model in which both soil and piles are discretized using BEM [15]. 

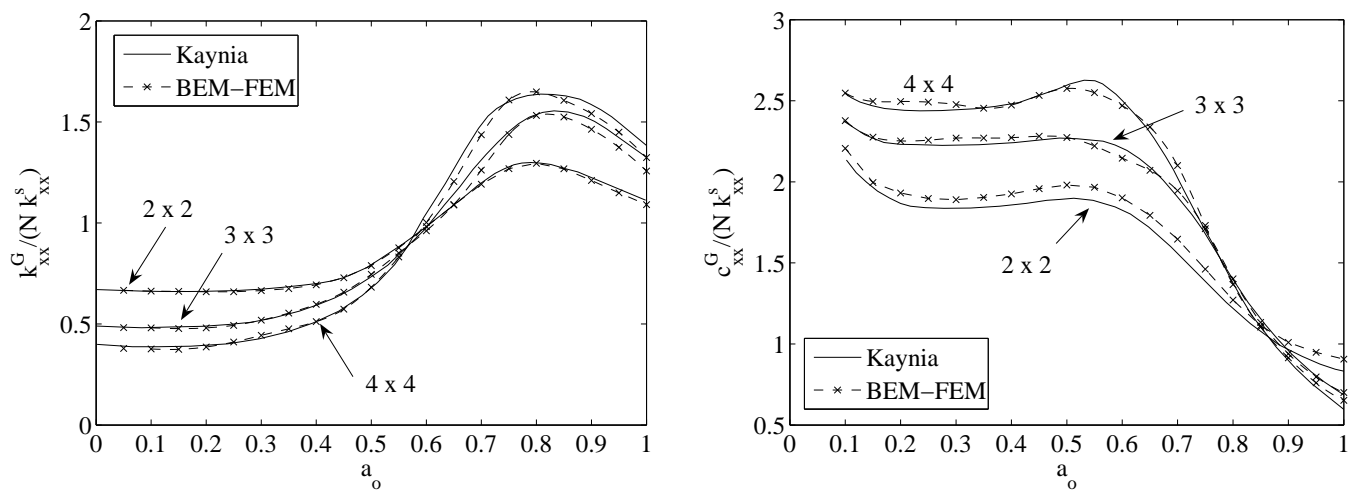

Figure 12: Horizontal impedances different pile groups embedded in a stiff soil with $\mathrm{s} / \mathrm{d}=5$. Comparison with Kaynia's solution.
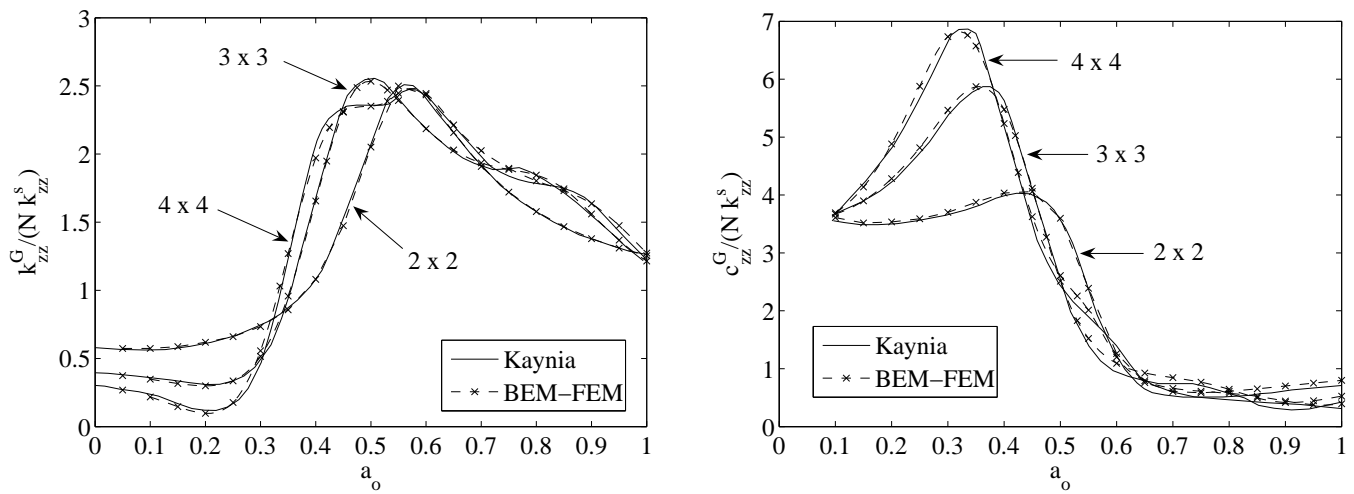

Figure 13: Vertical impedances different pile groups embedded in a stiff soil with $\mathrm{s} / \mathrm{d}=5$. Comparison with Kaynia's solution.
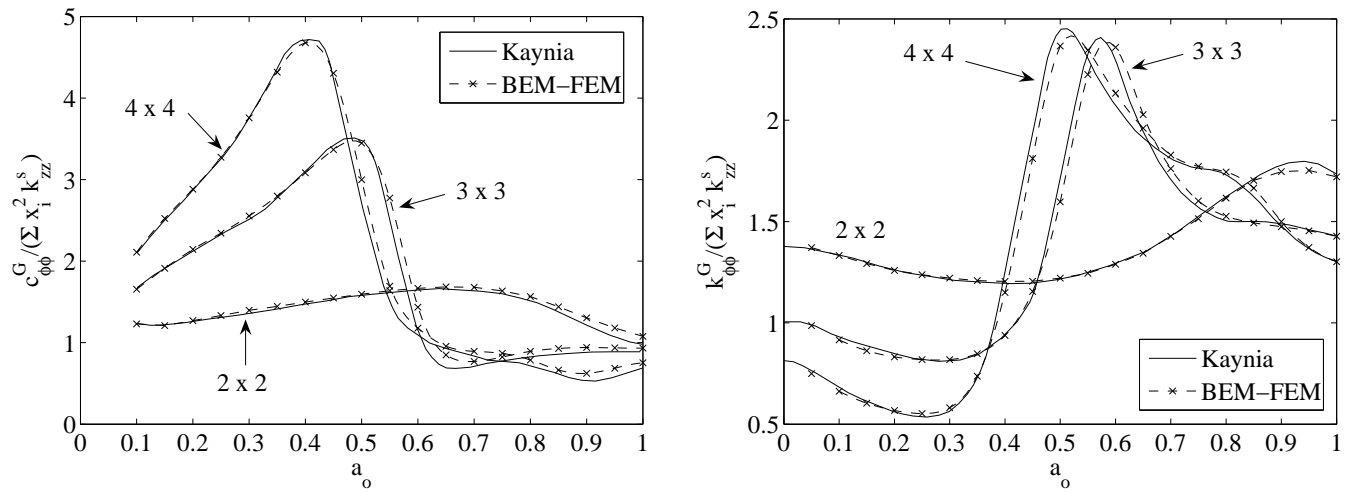

Figure 14: Rocking impedances different pile groups embedded in a stiff soil with $\mathrm{s} / \mathrm{d}=5$. Comparison with Kaynia's solution.

\section{Revision and conclusion}

In this paper, a three-dimensional BEM-FEM coupling model for the time-harmonic dynamic analysis of piles and pile groups embedded in homogeneous isotropic viscoelastic soils has been presented. 

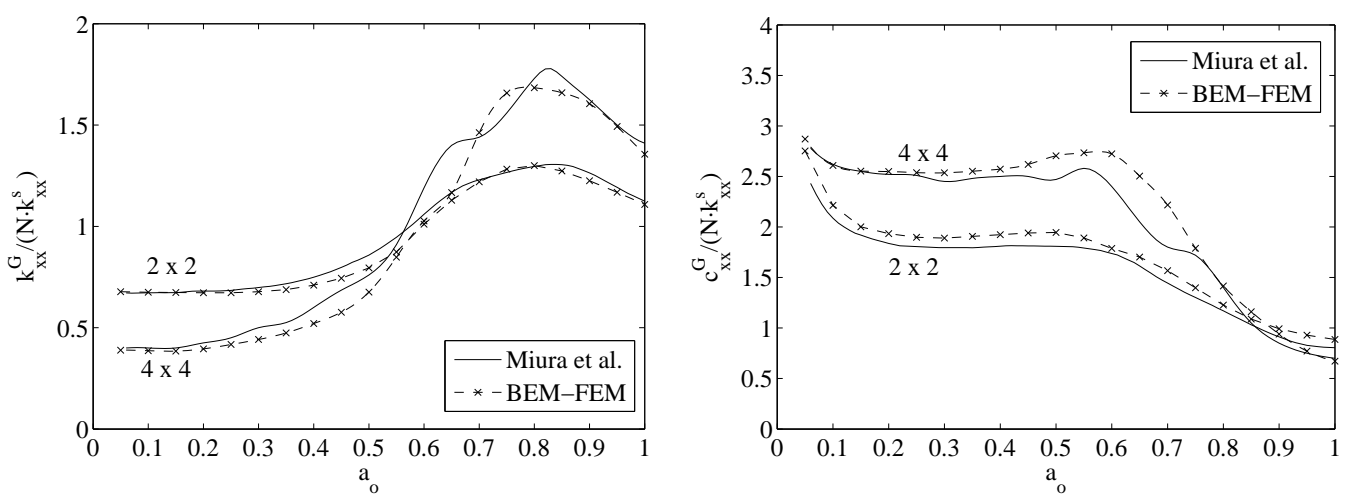

Figure 15: Horizontal impedances of $2 \times 2$ and $4 \times 4$ pile groups. Comparison with Miura's solution.
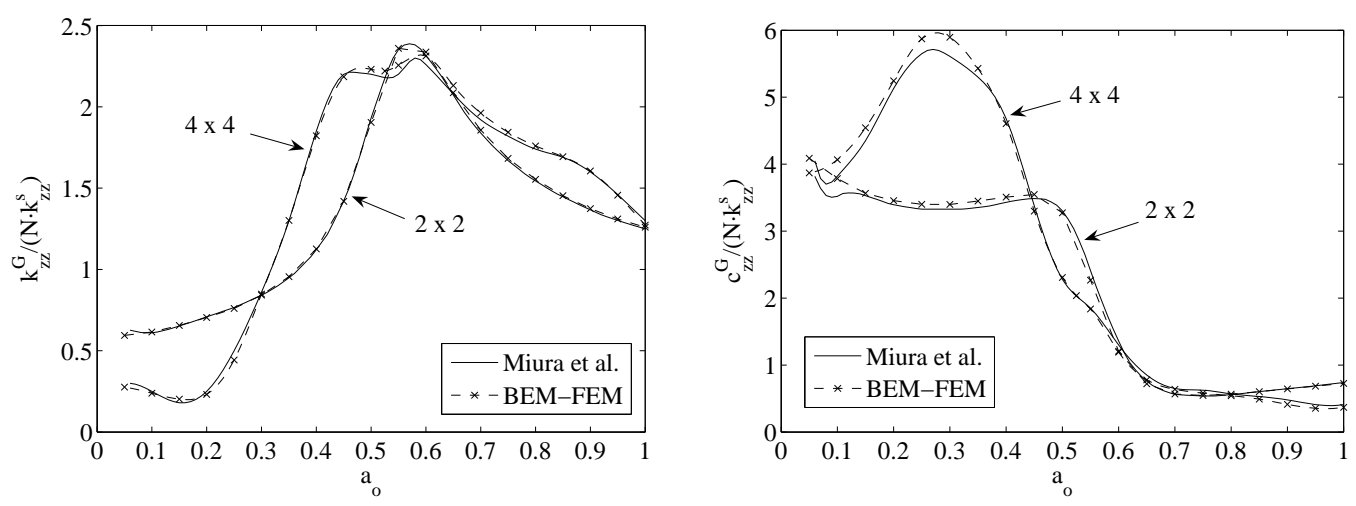

Figure 16: Vertical impedances of 2x2 and 4x4 pile groups. Comparison with Miura's solution.

(a) Shear forces due to horizontal excitation

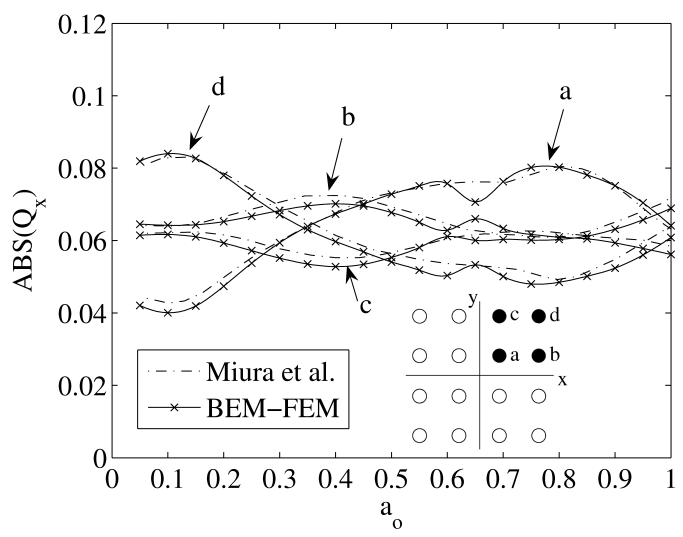

(b) Axial forces due to vertical excitation

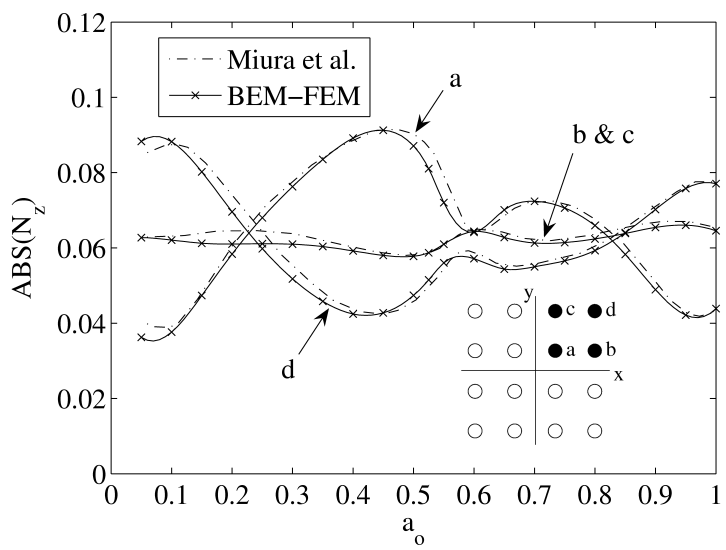

Figure 17: Forces on pile caps a $4 \times 4$ pile group

Piles are modelled using Finite Elements (FEM) as a beam according to the Bernoulli hypothesis, while the soil is modelled using Boundary Elements (BEM) as a continuum, semi-infinite, isotropic, homogeneous, linear, viscoelastic medium. In the soil equations, the presence of piles is replaced by internal punctual forces at the tip and load-lines placed along the pile axis, while it is assumed that 
the soil continuity is not altered by the presence of the piles. Then, the associated unknowns at internal points (displacements and tractions) are linked to piles variables through equilibrium and compatibility equations.

The main advantage of this model is the capacity of computing dynamic behaviour of piles with low computing times and low memory requirements in comparison to other methods that need to discretize the pile surface or volume. This way, pile groups with a big number of members can be analyzed without difficulty. Besides, once the surface (not necessarily flat) has been discretized, it does not need to be changed to analyze different sets of piles.

Several results of stiffness have been presented and compared to well known values taken from the literature, obtaining an excellent agreement. More cases than these presented in this work have been tested, all of them with the favourable conclusions. Although all results have been given for homogeneous soils, different configurations including soil strata and rigid rocky beds can be easily taken into account. Furthermore, other variables such as displacements or internal axial and shear forces and bending moments along the pile can be obtained without difficulty.

\section{Acknowledgements}

This work was supported by the Ministry of Education and Science of Spain through research project BIA2004-03955-C02-02. L.A. Padrón is recipient of the FPU research fellowship AP-2004-4858 from the Ministry of Education and Science of Spain. Authors would like to thank for this support.

\section{References}

[1] A.M. Kaynia, "Dynamic stiffness and seismic response of pile groups", Research Report R8303. Massachusetts Institute of Technology. Cambridge, Mass. 1982.

[2] R.S.Y. Pak, P.C. Jennings, "Elastodynamic response of the pile under transverse excitation", J Eng Mech, ASCE 1987;113(7):1101-16.

[3] R.K.N.D. Rajapakse, A.H. Shah, "On the longitudinal harmonic motion of an elastic bar embedded in an elastic half-space", Int J Solids Struct 1987;23(2):267-85.

[4] R.K.N.D. Rajapakse, A.H. Shah, "On the lateral harmonic motion of an elastic bar embedded in an elastic half-space", Int J Solids Struct 1987;23(2):287-303.

[5] A.M. Kaynia, E. Kausel, "Dynamics of piles and pile groups in layered soil media", Soil Dyn Earthq Eng 1991;10:386-401

[6] G. Gazetas, K. Fan, A.M. Kaynia, E. Kausel "Dynamic interaction factors for floating pile groups", J Geotech Eng, ASCE 1991;117:1531-48.

[7] A.M. Kaynia, M. Novak, "Response of pile foundations to Rayleigh waves and obliquely incident body waves", Earthq Eng Struct Dyn 1992:21;303-18.

[8] K. Miura, A.M. Kaynia, K. Masuda, E. Kitamura, Y. Seto, "Dynamic behaviour of pile foundations in homogeneous and non-homogeneous media", Earthq Eng Struct Dyn 1994;23:183-92.

[9] A.M. Kaynia, S. Mahzooni, "Forces in pile foundations under seismic loading", J Engng Mech, ASCE 1996:122,46-53. 
[10] H.B. Coda, W.S. Venturini, M.H. Aliabadi "A general 3D BEM/FEM coupling applied to elastodynamic continua/frame structures interaction analysis", Int J Numer Meth Eng 1999;46:695712 .

[11] H.B. Coda, W.S. Venturini, "On the coupling of 3D BEM and FEM frame model applied to elastodynamic analysis", Int J Solids Struct 1999;36:4789-804.

[12] S.E. Kattis, D. Polyzos, D.E. Beskos, "Vibration isolation by a row of piles using a 3-D Frequency domain BEM", Int J Numer Meth Eng 1999;46:713-28.

[13] S.E. Kattis, D. Polyzos, D.E. Beskos, "Modelling of pile wave barriers by effective trenches and their sreening effectiveness", Soil Dyn Earthq Eng 1999:18:1-10.

[14] F. Vinciprova, J.J. Aznárez, O. Maeso, G. Oliveto, "Interaction of BEM analysis and experimental testing on pile-soil systems.", in "Problems in structural identification and diagnostic: General aspects and applications.", C. Davini, E. Viola (Editors), Springer-Verlag, 195-227, 2003.

[15] O. Maeso, J.J. Aznárez, F. García, "Dynamic impedances of piles and groups of piles in saturated soils", Comput Struct 2005;83:769-82.

[16] A.V. Mendonça, J.B. de Paiva, "A boundary element method for the static analysis of raft foundations on piles", Eng Anal Boundary Elem 2000:24;237-47.

[17] A.V. Mendonça, J.B. Paiva, "An elastostatic FEM/BEM analysis of vertically loaded raft and piled raft foundation", Eng Anal Boundary Elem 2003:27;919-33.

[18] R. Matos Filho, A.V. Mendonça, J.B. Paiva, "Static boundary element analysis of piles submitted to horizontal and vertical loads", Eng Anal Boundary Elem 2005;29:195-203.

[19] R.W. Clough, J.Penzien, "Dynamics of structures", McGraw-Hill, 1982.

[20] J. Domínguez, "Boundary elements in dynamics", Southampton, New York, Computational Mechanics Publications \& Elsevier Applied Science; 1993.

[21] H.B. Li, G.M. Han, H.A. Mang, "A new method for evaluation singular integrals in stress analysis of solids by the Direct Boundary Element Method", Int J Numer Meth Eng 1985;21:2071-98.

[22] F. Chirino, O. Maeso, J.J. Aznárez, "Una técnica simple para el cálculo de las integrales en el sentido del valor principal en el MEC 3D", Revista Internacional de Métodos Numéricos para Cálculo y Diseño en Ingeniería 2000;16(1):77-95. 